\title{
Multivariate Statistical Analysis Uncovers Spectrum-Effect Relationship between HPLC Fingerprints and Antioxidant Activity of Saffron
}

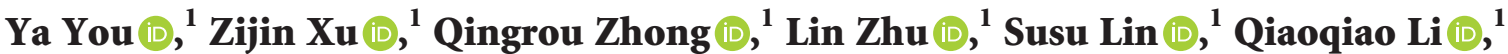 \\ Yifeng Cao $\mathbb{D}^{1},{ }^{1}$ Yi Tao $\mathbb{D}^{1},{ }^{1}$ Suhong Chen $\mathbb{D}^{2}{ }^{2}$ and Ping Wang ${ }^{1}{ }^{1}$ \\ ${ }^{1}$ College of Pharmacy, Zhejiang University of Technology, No. 18 Chaowang Road, Zhaohui District, Hangzhou 310000, China \\ ${ }^{2}$ Collaborative Innovation Center of Yangtze River Delta Region Green Pharmaceuticals, Zhejiang University of Technology, \\ No. 18 Chaowang Road, Zhaohui District, Hangzhou 310014, China
}

Correspondence should be addressed to Suhong Chen; chensuhong@aliyun.com and Ping Wang; wangping45@aliyun.com Received 19 August 2021; Revised 18 October 2021; Accepted 6 November 2021; Published 24 November 2021

Academic Editor: Eulogio J. Llorent Mart nez

Copyright (C) 2021 Ya You et al. This is an open access article distributed under the Creative Commons Attribution License, which permits unrestricted use, distribution, and reproduction in any medium, provided the original work is properly cited.

Crocus sativus L. is commonly used as functional food and medicinal herb in traditional Chinese medicine. In this study, the spectrum-effect relationship was established between HPLC fingerprints and in vitro antioxidant activity of saffron to improve the quality evaluation method of saffron. The fingerprints of 21 batches of saffron collected from different regions were assessed, and the data were further analyzed by chemometric methods, including similarity analysis, hierarchical clustering analysis, principal component analysis, and orthogonal partial least squares discriminant analysis. The spectrum-effect relationship between fingerprints and antioxidant effect of saffron was analyzed by grey relational analysis and partial least square methods to figure out the antioxidant component of saffron. Thirteen common peaks of 21 batches of saffron were included in the analysis, and peak 3 (picrocrocin), peak 7 (crocin I), and peak 10 (crocin II) were identified as the main active components responsible for antioxidant efficacy. Besides, a multi-index quality control method was developed for simultaneous determination of these three antioxidant components in saffron. Taken together, this study provided new strategies for the quality control and the development of new bioactive products of saffron in the future.

\section{Introduction}

Saffron is an expensive spice derived from the stigma of the Crocus sativus L., which has been mainly cultivated in Iran, Greece, Morocco, India, Spain, and Italy. About 70,000 Crocus sativus L. flowers are required to produce $1 \mathrm{~kg}$ dry saffron, which is the main reason for its high cost. In addition to being used as food and dye, saffron has many therapeutic properties. Previous studies have demonstrated that saffron has the biological activities of cardiovascular protection, liver protection, antidepression, anticancer, and anti-inflammatory and can be potentially used as a hypoglycemia agent and immune enhancer [1-7].

The scarcity of resources and high cost of saffron lead to the frequent occurrence of saffron adulteration in the market, such as plant-derived materials like Zea mays
L. (stigma), Chrysanthemum morifolium Ramat. (stigma), Carthamus tinctorius L. (stigma), corn silk, dyed corn stigma, turmeric, gardenia, rubia, calendula and artificial colorants like tartrazine, amaranth, sunset yellow, orange II, and new coccine [8-13]. The extracts of gardenia were added to saffron commonly because of the pigments in the extracts were similar to crocetin esters (crocins) present in saffron and thus could be concealed to a greater degree in the saffron $[14,15]$.

Adulteration of saffron in the market brings about attention of the quality control of saffron. The international standard ISO 3632-2011 for grading saffron reports a standard UV-Vis spectrophotometric method, which tests the strength of aroma, color, and flavour of saffron by determining the concentrations of safranal, crocin, and picrocrocin [16]. However, recent studies indicated that there 
was no correlation between the content of safranal and the UV absorbance value at $330 \mathrm{~nm}$ (the maximum UV absorption wavelength of safranal) according to the international standard ISO 2631-2011 [17]. Additionally, the standard UV-Vis method of ISO was used for grading saffron and may not reveal saffron adulteration with amounts up to $20 \%(\mathrm{w} / \mathrm{w})$ of safflower, turmeric, or calendula $[17,18]$. It can thus be seen that the grading of saffron depend on ISO method is not credible and cannot adequately distinguish between genuine and adulterated saffron.

Many published studies have focused on the quality standard of saffron, and various analytical techniques were applied to the quality control of saffron, such as high-performance liquid chromatography (HPLC), gas chromatography (GC), near-infrared spectroscopy (NIRS), ultra-highperformance liquid chromatography coupled to tandem mass spectrometry (UHPLC-MS/MS), and electronic nose (E-Nose) [19-23]. For the authentication of saffron, different analytical methods, including Raman spectroscopy, optical nanosensor, gas chromatography with mass spectrometry detection (GC-MS), microchip electrophoresis (MCE), headspace flash gas chromatography with flame ionization detection (HS-GC-FID), and nuclear magnetic resonance (NMR) spectroscopy, were assessed [16, 24-27]. These techniques were conducive to the determination of different components of saffron, especially crocin. However, few studies focused on the correlation between quality evaluation and biological activities of saffron.

Spectrum-effect relationships have been widely used to screen the active compounds of TCMs by combining chromatographic fingerprint of TCMs with their biological activity. Chromatographic fingerprints of traditional Chinese medicines (TCMs) contain a large number of information and could express the chemical characteristics of samples integrally $[28,29]$. In recent years, spectrum-effect relationships were often being used to assess the quality control of TCMs [30]. For example, chlorogenic acid and 3,4-dicaffeoylquinic acid from Lonicerae Japonicae Flos and Lonicerae Flos were selected as the major antibacterial components by spectrum-effect relationships [31]. Menthone, isomenthone, pulegone, piperitone, and $\beta$-caryophyllene were identified as the dominant constituents responsible for the antioxidant and anti-inflammatory activities of S. tenuifolia essential oil [32]. In Chinese Pharmacopoeia (2020 edition), the contents of picrocrocin were newly used as the quality control standard for saffron, coupled with the content of crocins I and II. However, the relationship between these components and bioactivity requires further investigation to improve the quality control standard of saffron.

In this study, HPLC was used to establish the fingerprints of 21 batches of saffron. Then, similarity analysis (SA), hierarchical clustering analysis (HCA), principal component analysis (PCA), and orthogonal partial least squares discriminant analysis (OPLS-DA) were applied to distinguish differences among the 21 batches of saffron. Subsequently, the antioxidant activity was evaluated by 1,1-diphenyl-2picrylhydrazyl (DPPH) radical-scavenging assay and hydroxyl $(\bullet \mathrm{OH})$ radical-scavenging assay. The spectrumeffect relationship between HPLC fingerprints and antioxidant activities were elucidated by grey relational analysis (GRA) and partial least square (PLS) analysis. The potential active compounds of saffron were discovered. Finally, a quantitative method for the determination of the potential active compounds, crocin I, crocin II, and picrocrocin, was developed.

\section{Experimental}

2.1. Materials and Reagents. Twenty-one batches of saffron were collected from different regions, as shown in Table 1. These were obtained directly from the producers and packers with a guarantee of their origin and freedom from fraud. All samples were authenticated as plant of Crocus sativus L., and voucher specimens were deposited in the herbarium of Zhejiang University of Technology.

Picrocrocin (97.6\% purity) standard reference substance was purchased from the National Institutes for Food and Drug Control (Beijing, China). Crocin I ( $\geq 98.0 \%$ purity) and crocin II ( $\geq 98.0 \%$ purity) standard reference substances were purchased from Shanghai Standards Technology Co., Ltd. (Shanghai, China). 2,2'-diphenyl-1-picrylhydrazyl (DPPH) was purchased from Sinopharm Chemical Reagent Co., Ltd. (Shanghai, China). Acetonitrile (HPLC grade) and methanol (HPLC grade) were purchased from Fisher Scientific (Pittsburgh, PA, USA). Purified water (Wahaha Purified Water) was purchased from Hangzhou Wahaha group Co., Ltd. (Hangzhou, China). All other reagents were of analytical grade.

\subsection{Preparation of Samples and Reference Substance Solutions.} All the saffron samples used in this study were dried samples. The sample drying method was in accordance with the low temperature drying $\left(<60^{\circ} \mathrm{C}\right)$ stipulated in technical regulation for production of saffron crocus (Crocus sativus L.) (NO. DB 33/T 530-2014, 2014 Version). The sample solutions were prepared according to the method of ISO 3632$2011.50 \mathrm{mg}$ of grounded saffron was accurately weighed and quantitatively transferred to a $50 \mathrm{~mL}$ volumetric flask and diluted to scale with $40 \mathrm{~mL} \mathrm{50 \%} \mathrm{ethanol.} \mathrm{Ultrasonic} \mathrm{was}$ carried out at $50^{\circ} \mathrm{C}$ for $10 \mathrm{~min}$ and kept away from light. Then, 50\% ethanol was added to a constant volume and tightened. The volumetric flask was shaken evenly. The sample solution was filtered through $0.45-\mu \mathrm{m}$ filter membrane before use.

All reference substances were weighed to obtain $1 \mathrm{mg} /$ $\mathrm{mL}$ picrocrocin, crocin I, and crocin II stock solutions. An appropriate amount of stock solution was taken and diluted to an appropriate concentration with $50 \%$ ethanol. The solution was filtered using $0.45-\mu \mathrm{m}$ filter membrane before sample injection.

\subsection{HPLC Conditions of Fingerprint and Quantitative} Analysis. An Agilent 1260 HPLC system (Agilent Technologies Inc., California, USA) was used to detect the analytes. Chromatographic separation was carried out with 
TABLE 1: The origin and collection sites of 21 batches of saffron used in this study.

\begin{tabular}{|c|c|c|c|c|c|}
\hline ID & Origin & Collection site & ID & Origin & Collection site \\
\hline S1 & Zhejiang, China & Jiande Saffron Agricultural Cooperative & S12 & Tibet, China & Bozhou Yonggang Slices Factory co. LTD \\
\hline S2 & Zhejiang, China & Jiande Saffron Agricultural Cooperative & S13 & Shanghai, China & Bozhou Kangmei TCMs Market \\
\hline S3 & Zhejiang, China & Jiande Saffron Agricultural Cooperative & S14 & Iran & Bozhou Kangmei TCMs Market \\
\hline S4 & Henan, China & Dancheng Saffron Agricultural Cooperative & S15 & Iran & Bozhou Kangmei TCMs Market \\
\hline S5 & Zhejiang, China & Jiande Saffron Agricultural Cooperative & S16 & Iran & Bozhou Kangmei TCMs Market \\
\hline S6 & Anhui, China & Bozhou Kangmei TCMs Market & S17 & Tibet, China & Bozhou Kangmei TCMs Market \\
\hline S7 & Anhui, China & Bozhou Kangmei TCMs Market & S18 & Tibet, China & Bozhou Kangmei TCMs Market \\
\hline S8 & Anhui, China & Bozhou Kangmei TCMs Market & S19 & Iran & Bozhou Kangmei TCMs Market \\
\hline S9 & Anhui, China & Bozhou Kangmei TCMs Market & S20 & Iran & Bozhou yonggang Slices Factory co. LTD \\
\hline S10 & Anhui, China & Bozhou yonggang Slices Factory co. LTD & S21 & Henan, China & Dancheng Saffron Agricultural Cooperative \\
\hline S11 & Dubai & Bozhou Kangmei TCMs Market & & & \\
\hline
\end{tabular}

an Eclipse XDB-C18 column $(4.6 \mathrm{~mm} \times 150 \mathrm{~mm}, 5 \mu \mathrm{m}$, Agilent Technologies Inc., California, USA) using the mobile phase composed of methanol (A), acetonitrile (B), and $0.2 \%$ formic acid (C) with the following gradient elution: 0-60 min, 10\%-100\% A; 13.5\%-0 B; 76.5\%-0 C, $1 \mathrm{~mL} \cdot \mathrm{min}^{-1}$ flow rate, $20 \mu \mathrm{L}$ volume injection, and column set at $35^{\circ} \mathrm{C}$. The detection wave length was 257 and $440 \mathrm{~nm}$.

\subsection{Antioxidant Activities}

2.4.1. DPPH Radical-Scavenging Assay. $3 \mathrm{~mL}$ of different concentrations of sample solutions and $70 \mu \mathrm{g} / \mathrm{mL} \mathrm{DPPH}$ solution were mixed in the test tube. The mixture was kept at $25^{\circ} \mathrm{C}$ in darkness for $30 \mathrm{~min} .3 \mathrm{~mL}$ of $50 \%$ ethanol and DPPH solution were mixed as a control. And $3 \mathrm{~mL}$ of sample solutions and ethanol were mixed as a blank. The absorbance of each reaction mixture was measured at $517 \mathrm{~nm}$. The $\mathrm{DPPH}$ radical-scavenging capacity and $\mathrm{IC}_{50}$ were calculated of each sample.

$$
\text { DPPH } \%=1-\frac{\left(A_{\text {sample }}-A_{\text {blank }}\right)}{A_{\text {control }}} \times 100 \% .
$$

2.4.2. $\cdot O H$ Radical-Scavenging Assay. $2 \mathrm{~mL}$ of different concentrations sample solutions, $2 \mathrm{~mL}$ of $3 \mathrm{mmol} / \mathrm{L}$ salicylic acid solution, and $2 \mathrm{~mL}$ of $3 \mathrm{mmol} / \mathrm{L} \mathrm{FeSO}_{4}$ solution were mixed in the test tube. Subsequently, $2 \mathrm{~mL}$ of $3 \mathrm{mmol} / \mathrm{L}$ $\mathrm{H}_{2} \mathrm{O}_{2}$ was added to start the Fenton reaction, and the mixture was incubated for $30 \mathrm{~min}$ at $37^{\circ} \mathrm{C} .2 \mathrm{~mL}$ of $50 \%$ ethanol was used to instead of sample solution as a control, and $2 \mathrm{~mL}$ of $\mathrm{H}_{2} \mathrm{O}$ was used instead of $3 \mathrm{mmol} / \mathrm{L} \mathrm{H}_{2} \mathrm{O}_{2}$ as a blank. The absorbance of each reaction mixture was measured at $510 \mathrm{~nm}$. The $\cdot \mathrm{OH}$ radical-scavenging capacity and $\mathrm{IC}_{50}$ were calculated of each sample.

$$
\text { . OH } \%=1-\frac{\left(A_{\text {sample }}-A_{\text {blank }}\right)}{A_{\text {control }}} \times 100 \% .
$$

2.5. Statistical Analysis. Data analysis was performed by the software Similarity Evaluation System for Chromatographic Fingerprint of Traditional Chinese Medicine (Version 2012). HCA was performed by SPSS (Version 24). PCA, PLS, and
OPLS-DA were performed by SIMCA-P (Version 14.0). Grey relational degree was performed by DPS. Quantitation of three marker components were calculated based on the calibration curves, and the results were expressed as mean \pm SD.

\section{Results and Discussion}

3.1. Optimization of HPLC Conditions. The preparation methods of the sample were first studied according to the stirring method in ISO-3632-2011 and the ultrasonic extraction method in our previous study $[19,33]$. The results showed that there was no significant difference between the two extraction methods. Considering the stability of sample solution and the convenience of experiment, the ultrasonic extraction method was selected to prepare sample solution. To obtain excellent HPLC fingerprint, various parameters including extraction solvent (water, 25\%, 50\%, 75\%, and $100 \%$ of methanol, $25 \%, 50 \%, 75 \%, 100 \%$ of ethanol, $v / v)$, extraction time $(10 \mathrm{~min}, 20 \mathrm{~min}, 30 \mathrm{~min}, 40 \mathrm{~min}, 50 \mathrm{~min}$, $60 \mathrm{~min}$ ), light (on light or away from light), and extraction solid-liquid ratio $(1: 0.5,1: 1,1: 5,1: 20, w / v)$ were optimized. The results showed that $50 \%$ of ethanol and $1: 1$ of solid-liquid ratio provided better extraction efficiency and chromatographic separation (Figure 1). Furthermore, dual wavelengths ( $257 \mathrm{~nm}$ and $440 \mathrm{~nm}$ ) have been proved to provide more detection peaks, stronger UV absorption, and better peak shape, so these two wavelengths were selected as the detection wavelengths in the HPLC analysis of fingerprint.

3.2. HPLC Fingerprint Analysis and Similarity Analysis. Recently, fingerprints combined with multivariate statistical analysis have been used to classify and discriminate different TCMs sources successfully [34]. In the current study, the spectrum of sample S4 was used as the reference spectrum, and the representative HPLC fingerprints of 21 batches of saffron were established as shown in Figures 2(a) and 2(b). The generated representative fingerprints were showed in Figures 2(c) and 2(d). The RT and PA information of 13 common peaks in the fingerprint were extracted, and the RT and characteristic absorption wavelength of the standard reference substances were compared. The following results were obtained: peak 3 was picrocrocin, peak 7 was crocin I, 

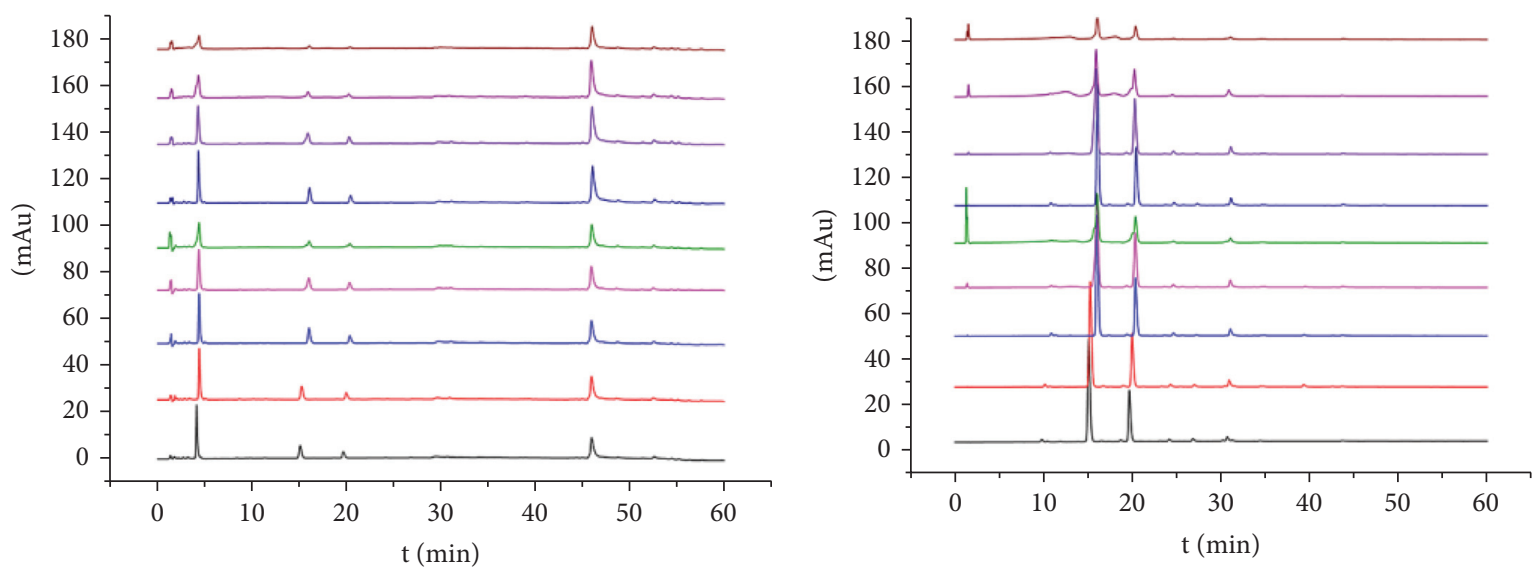

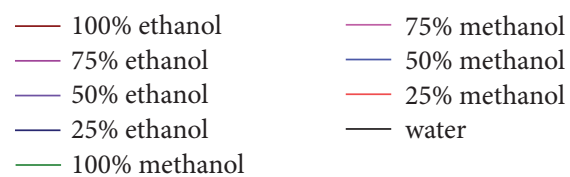

(a)
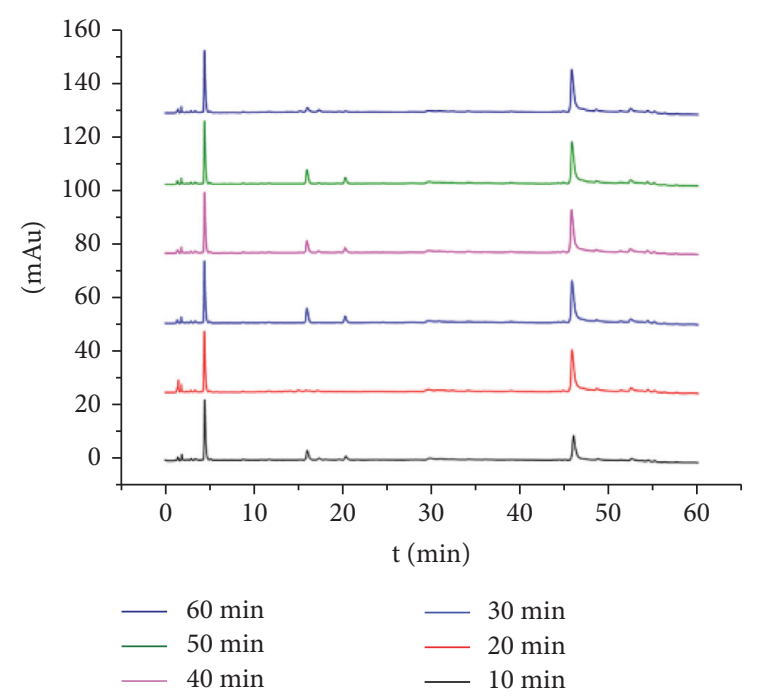

(c)

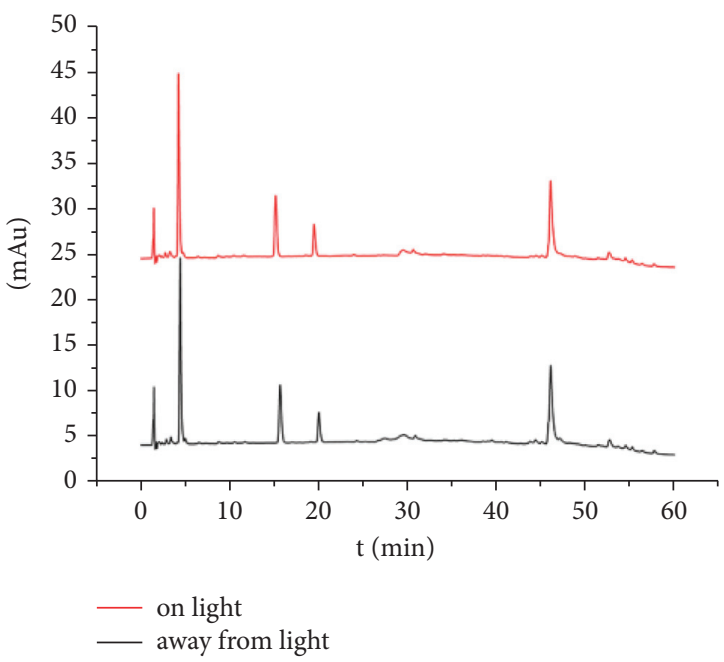

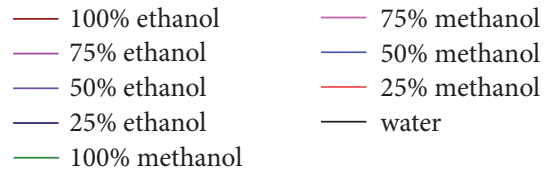

(b)

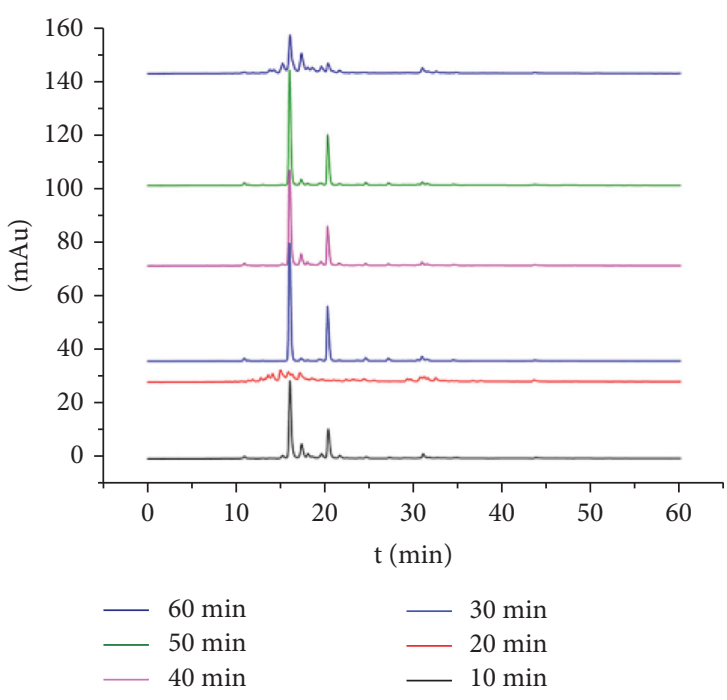

(d)

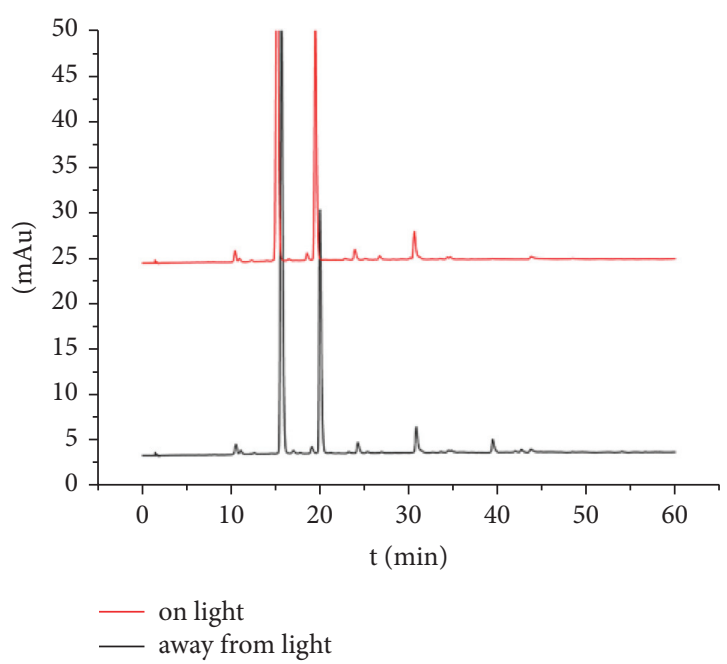

(f)

Figure 1: Continued. 


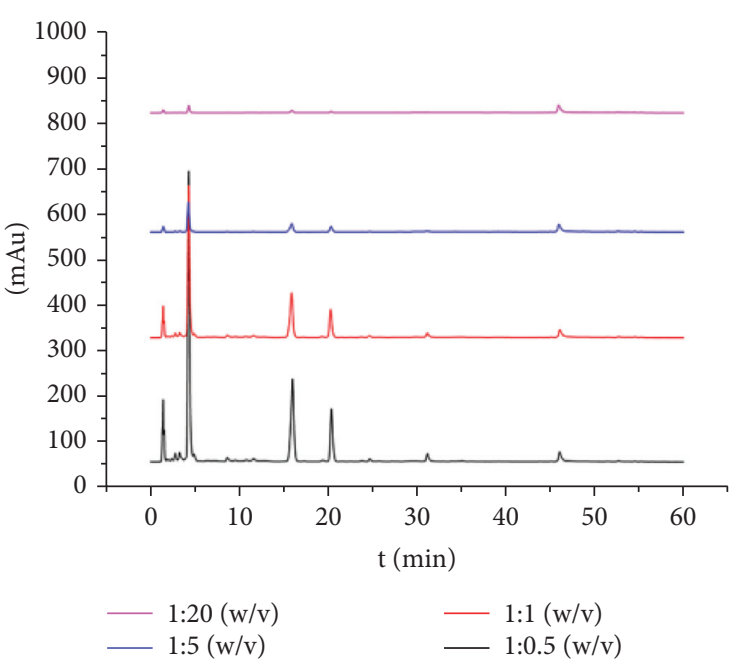

(g)

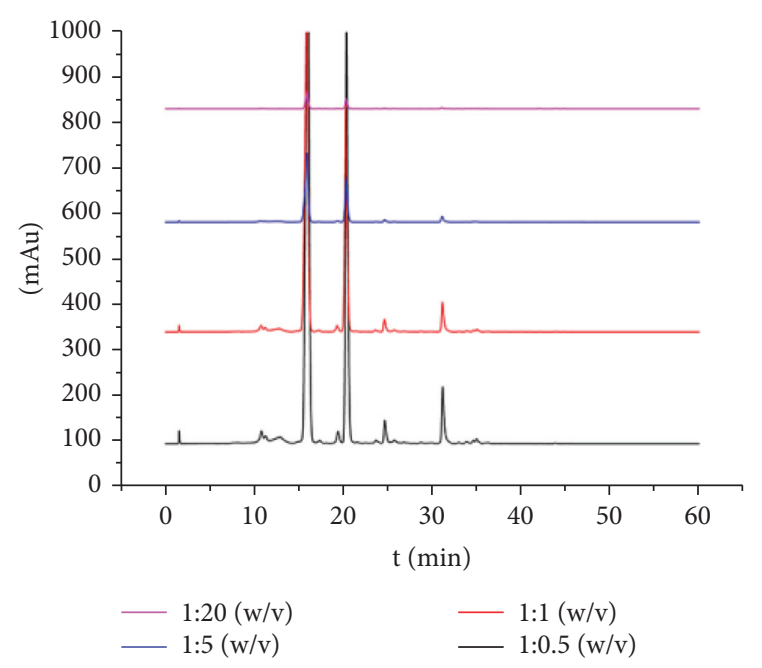

(h)

Figure 1: Effect of extraction solvent $(a, b)$, extraction time $(c, d)$, light $(e, f)$, and solid-liquid ratio $(g, h)$ on the HPLC fingerprints of saffron at $257 \mathrm{~nm}(\mathrm{a}, \mathrm{c}, \mathrm{e}, \mathrm{g})$ and $440 \mathrm{~nm}(\mathrm{~b}, \mathrm{~d}, \mathrm{f}, \mathrm{h})$.

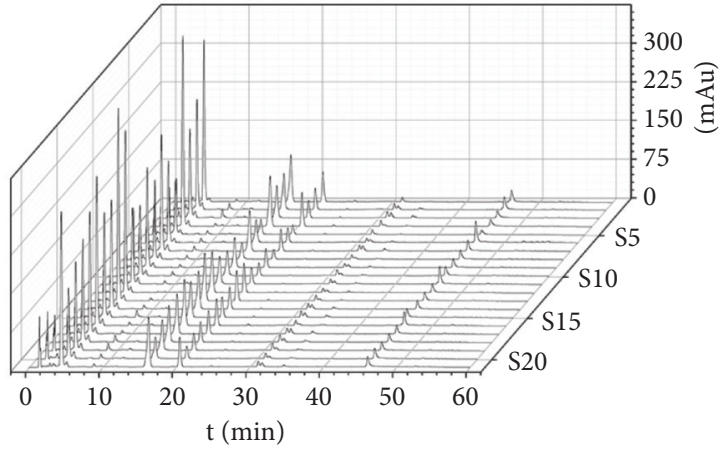

(a)

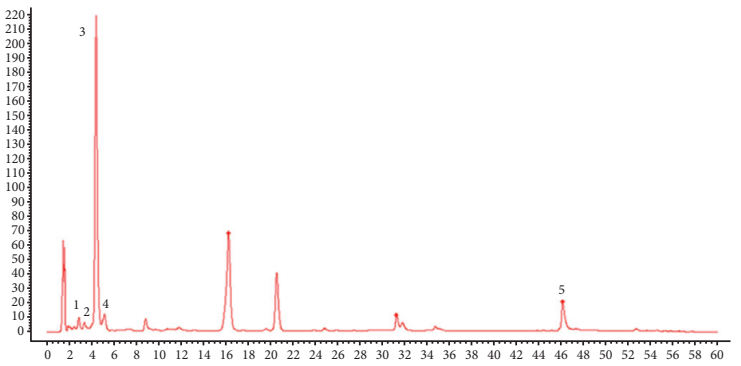

(c)

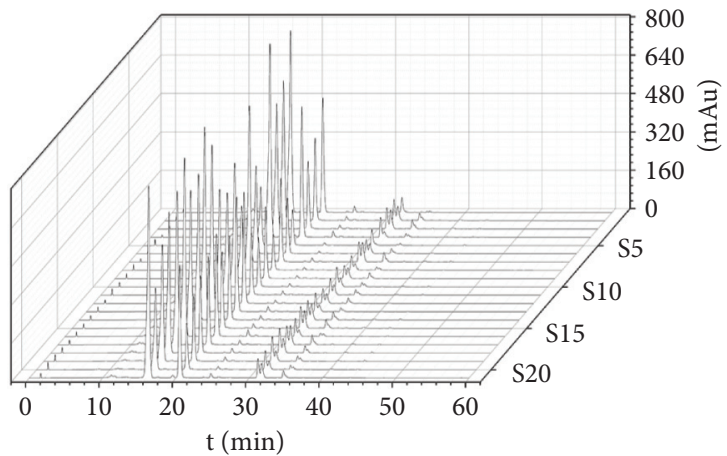

(b)

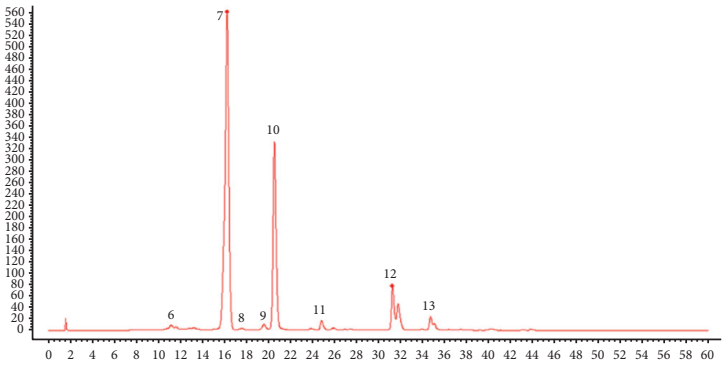

(d)

FigURE 2: HPLC fingerprints of 21 batches of saffron at $257 \mathrm{~nm}$ (a) and $440 \mathrm{~nm}$ (b) as well as representative fingerprint of saffron at $257 \mathrm{~nm}$ (c) and $440 \mathrm{~nm}(\mathrm{~d})$.

peak 10 was crocin II, peak 12 was crocin III, and peak 13 was cis-crocin I.

In this study, the similarity calculation was carried out by Similarity Evaluation System for Chromatographic Fingerprint of Traditional Chinese Medicine software (Version 2012) issued by Chinese Pharmacopoeia Committee. Time window width was set as 0.1 , average mode was used, and then, all the samples had multipoint correction and automatic match to generate a representative fingerprint that represented the characteristic mode. The similarity was calculated by comparing chromatograms of saffron with the representative fingerprints $[35,36]$. The results showed that the similarity of 21 batches of saffron was $0.962-0.998$ at $257 \mathrm{~nm}$ and $0.992-1$ at $440 \mathrm{~nm}$. The similarity of S1 to S21 at $257 \mathrm{~nm}$ was $0.996,0.998,0.993$, $0.993,0.962,0.981,0.997,0.976,0.998,0.994,0.987,0.993$, $0.993,0.997,0.998,0.998,0.998,0.997,0.996,0.988$, and 0.996 , respectively; the similarity of S1 to S21 at $440 \mathrm{~nm}$ was $0.997,1,0.998,0.997,0.992,0.999,0.998,0.998,0.999$, $0.998,1,0.997,0.999,1,0.999,0.999,0.999,0.999,0.999$, 
TABLE 2: The regression equation, $R^{2}$, linear range, and standard errors.

\begin{tabular}{lccccc}
\hline Name & Regression equation & \multirow{2}{*}{$R^{2}$} & Linear range $(\mu \mathrm{g} / \mathrm{mL})$ & \multicolumn{2}{c}{ Standard errors } \\
Slope & $18.92 \sim 302.70$ & 0.12 & 19.24 \\
Crocin I & $y=95.537 \times+43.146$ & 0.9999 & $6.28 \sim 100.50$ & 0.16 & 8.51 \\
Crocin II & $y=139 \times-5.9062$ & 0.9999 & $17.78 \sim 284.40$ & 0.02 \\
Picrocrocin & $y=28.282 \times-0.974$ & 0.9999 & 2.57 \\
\hline
\end{tabular}

0.998 , and 1 , respectively. The high similarity $(\geq 0.962)$ indicated that the quality of saffron was generally stable and the established fingerprint method could be used for the identification and quality control of saffron.

\subsection{Method Validation}

3.3.1. Method Validation for the HPLC Fingerprint Analysis. The method validation results of the HPLC fingerprint showed that the relative standard deviations (RSDs) of precision, repeatability, and stability of the retention time (RT) and the peak area (PA) met the prescribed requirements. The variations in the RT of the characteristic peaks were less than $0.5 \%$, and the variations in the PA are less than $3.0 \%(n=6)$.

3.3.2. Method Validation of Quantitative Analysis. The method of quantitative analysis was validated in terms of linearity of calibration curves, precision, stability, repeatability, and recovery. Taking the sample concentration as the abscissa $(x, \mathrm{mg} / \mathrm{L})$ and PA as the ordinate $(y)$, the linear regression analysis was carried out. The regression equation, $R^{2}$, linear range, and standard errors were shown in Table 2. The results indicated that the linear relationship obtained for each target compound was reliable, and the obtained calibration curves were suitable for HPLC analysis.

The precision, stability, and repeatability were assessed by the PA of P3, P7, and P10, respectively. The RSDs of precision of crocin I, crocin II, and picrocrocin were $1.11 \%$, $0.10 \%$, and $0.10 \%$, respectively, the RSDs of stability were $1.31 \%, 0.47 \%$, and $0.21 \%$, respectively, and the RSDs of repeatability were $1.60 \%, 1.26 \%$, and $0.21 \%$, respectively. Recovery was measured by the standard addition method. The sample (S4) was added with high, medium, and low levels of a mixed standard solution of the three compounds in triplicate. The average recovery rates of crocin I; crocin II; , and picrocrocin were $99.58 \%, 98.18 \%$, and $100.04 \%$, respectively. All the results of the method validation tests demonstrated that the proposed method was reliable and valid.

3.4. HCA. In this study, SPSS statistical software (Version 24.0) was used to perform HCA on the fingerprint of 21 batches of saffron, using the square Euclidean distance as the interval and using the intragroup linkage method, as shown in Figure 3. According to the results of HCA, 21 batches of saffron could be divided into two categories: S5, S6, S8, S9, S10, S11, S19, and S20 were in one category, while S1-S4, S7, S12-S18, and S21 were in the other category. In order to evaluate the difference between the two categories of saffron, PCA and OPLS-DA were used to the further analysis.

3.5. PCA and OPLS-DA. PCA is a multivariate statistical method which converts multiple variables into a few unrelated comprehensive variables. The purpose of PCA is to remove overlapping information among numerous of information by dimensionality reduction [37]. The PA of 13 common peaks was inputted as the variables. Then, the PCA scoring map was obtained by SIMICA-P software, which divided saffron into two categories: S2, S3, S5, S6, S8, S9, S10, S11, S19, and S20 were assigned to one category, while S1, S4, S7, S12-S18, and S21 were in another category (Figure 4(a)). SPSS was used for PCA analysis to obtain principal components (PCs). According to the principle of screening PCs in PCA, the first three PCs were identified with eigenvalue $\lambda>1$, of which the cumulative contribution rate was $77.515 \%$ (Table 3). Thereby, the three PCs can be used for the evaluation of saffron quality [30,38]. Among the three PCs, the cumulative contribution rate of the PC1 was $47.932 \%$, which represented the highest influence to the quality control of saffron [39]. This information primarily originated from $\mathrm{P} 2, \mathrm{P} 3$, and $\mathrm{P} 6-\mathrm{P} 11$, while $\mathrm{P} 4, \mathrm{P} 9, \mathrm{P} 12$, and $\mathrm{P} 13$ showed the high loading values to the PC2. P2, P5, and P13 showed the high loading value to the PC3 (Table 4). These results showed that the quality difference of saffron was affected by various components, but not by a single one.

The predictive ability parameter $Q^{2}$ was 0.326 . Based on previous study, the range of $Q^{2}$ from 0.3 to 0.4 indicated that the model had poor predictive power, which might be caused by the within-class divergence of individual groups [40]. It was with these considerations in mind that the OPLS-DA was further performed to extend a regression of the PCA because OPLS-DA had better discriminant ability for the samples with larger within-class divergence than PCA [41].

In OPLS-DA, the corresponding model was obtained by using the PA of 13 common peaks of saffron as input variable. The results were shown in Figures 4(b) and 4(c). In the OPLSDA model, the cumulative explanatory ability parameters $R_{X}^{2}$ and $R_{Y}^{2}$ were 0.944 and 0.781 , the predictive ability parameters $Q^{2}$ were 0.726 , and $R^{2}$ and $Q^{2}$ were all greater than 0.5 , which indicated that the model had certain stability and reliability and could be used to evaluate and distinguish different batches of saffron [42]. According to the OPLS-DA score, saffron from different origins were divided into two categories, which was similar to the results of HCA and PCA analysis. Statistical significance markers were selected according to the VIP predicted value in the model. Within the confidence interval of 0.95 , VIP $>1.0$ was selected as the 


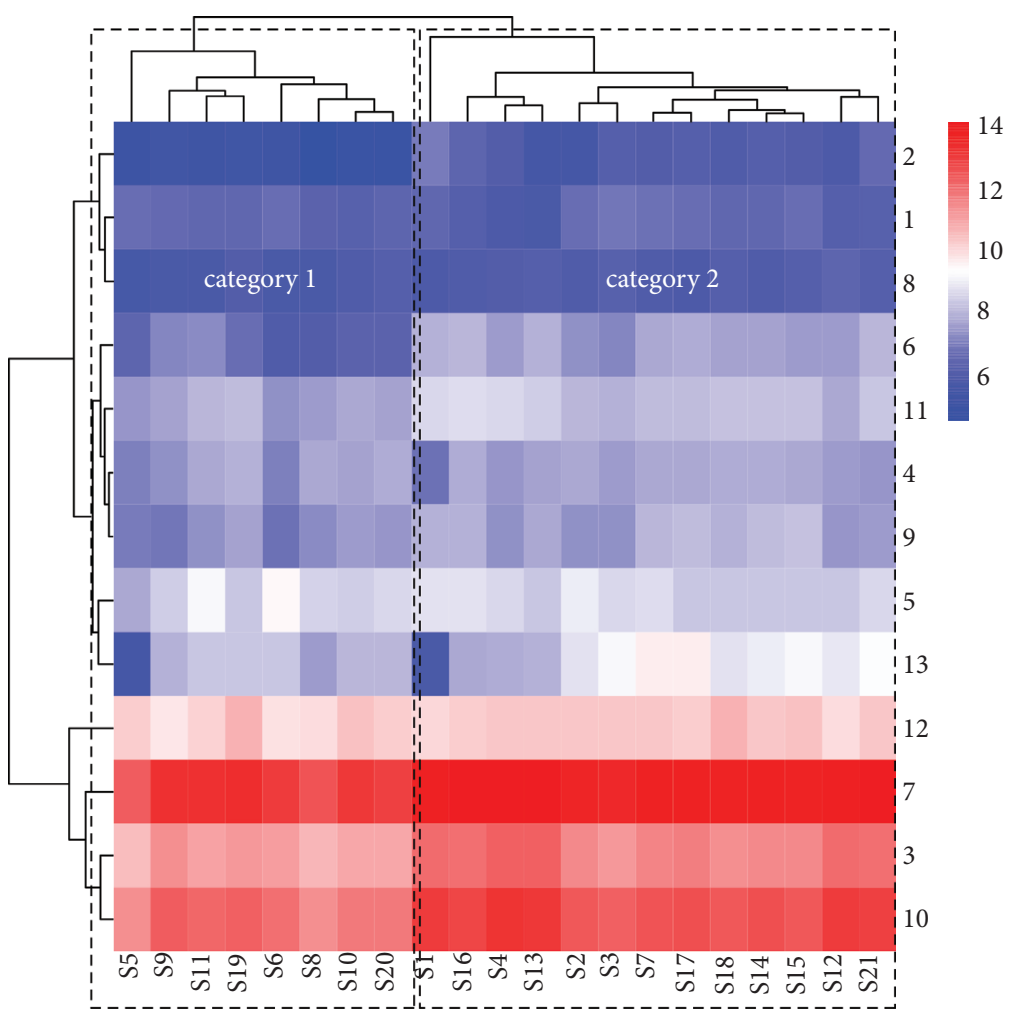

Figure 3: Heat map of the HCA of 21 batches of saffron.

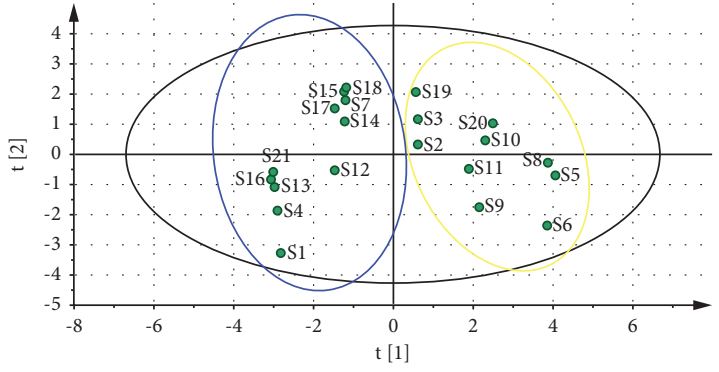

$\mathrm{R} 2 \mathrm{X}[1]=0.464 \quad \mathrm{R} 2 \mathrm{X}[2]=0.188 \quad$ Ellipse: Hotelling's T2 (95\%)

(a)

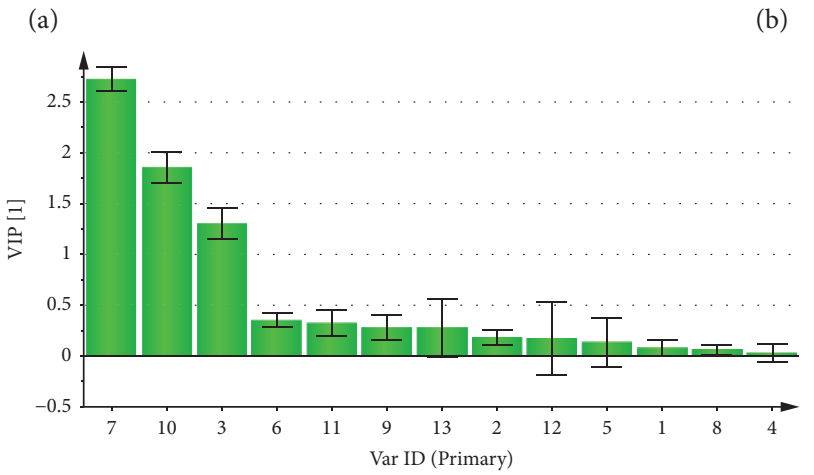

(c)

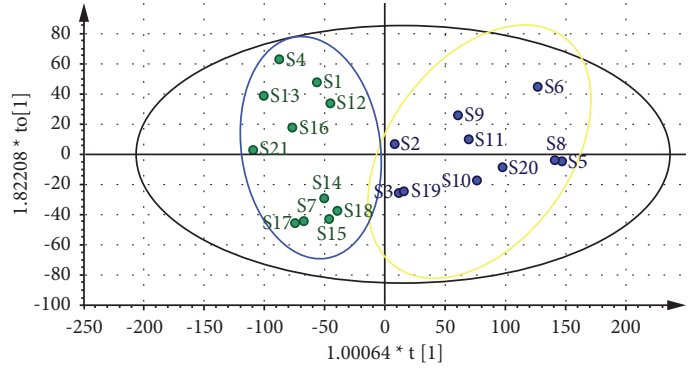

R2X [1] $=0.817 \quad$ R2Xo [1] $=0.127 \quad$ Ellipse: Hotelling's T2 (95\%)

- 1

) characteristic peaks (c). 
TABLE 3: Eigenvalue and variance cumulative contribution rate.

\begin{tabular}{lccc}
\hline Principal component & Eigenvalue $\lambda$ & Variance contribution rate (\%) & Cumulative variance contribute rate (\%) \\
\hline 1 & 6.231 & 47.932 & 47.932 \\
2 & 2.330 & 17.924 & 65.856 \\
3 & 1.516 & 11.659 & 77.515 \\
4 & 0.979 & 7.534 & 85.049 \\
5 & 0.822 & 6.321 & 91.369 \\
6 & 0.550 & 4.228 & 95.597 \\
7 & 0.243 & 1.872 & 97.469 \\
8 & 0.158 & 1.212 & 98.681 \\
9 & 0.095 & 0.729 & 99.410 \\
10 & 0.057 & 0.441 & 99.851 \\
11 & 0.010 & 0.074 & 99.925 \\
12 & 0.007 & 0.052 & 99.977 \\
13 & 0.003 & 0.023 & 100.000 \\
\hline
\end{tabular}

TABle 4: Component matrixes.

\begin{tabular}{lccc}
\hline Peak number & \multicolumn{3}{c}{ Principal component } \\
\hline 1 & 1 & 2 & 3 \\
2 & -0.392 & 0.397 & 0.743 \\
3 & 0.798 & -0.202 & 0.400 \\
4 & 0.897 & -0.350 & -0.129 \\
5 & 0.181 & 0.804 & -0.289 \\
6 & -0.043 & -0.271 & 0.551 \\
7 & 0.940 & -0.064 & 0.181 \\
8 & 0.981 & -0.119 & 0.012 \\
9 & 0.558 & 0.307 & -0.311 \\
10 & 0.702 & 0.461 & 0.169 \\
11 & 0.930 & -0.289 & -0.049 \\
12 & 0.887 & -0.098 & -0.009 \\
13 & 0.393 & 0.694 & -0.202 \\
\hline
\end{tabular}

quality control marker. The VIP values of P3 (crocin I), P7 (crocin II), and P10 (picrocrocin) were 2.72, 1.85, and 1.30, respectively. Therefore, crocin I, crocin II, and picrocrocin were identified as the quality control markers of saffron.

Permutation test, a computer-based resampling method for remodeling and predicting, was widely used in the computation of variable importance and confidence intervals $[43,44]$. It could be considered that the model had not been overfitted when the $Y$-axis intercept of $R^{2}$ and $Q^{2}$ for the established OPLS-DA models was less than 0.3 and 0.05 , respectively [45-47]. After 200 permutations, the intercepts of $R^{2}$ and $Q^{2}$ were 0.143 and -0.429 , respectively, which indicated that the established OPLS-DA model was reliable and not overfitted (Figure 5).

In Iranian pharmacopoeia and European pharmacopoeia, the quality control of saffron does not include quantitative analysis of chemical composition, while Japanese pharmacopoeia and Korean pharmacopoeia list the sum of crocin I and crocin II for the quality control of saffron for only qualitative analysis. Many literatures have shown that the crocins play a critical role in quality control of saffron. Additionally, a variety of components, including crocins, picrocrocin, crocetin, and safranal, show unique pharmacological activity $[10,48,49]$. To sum up, the picrocrocin, crocin I, and crocin II were identified as the quality control of saffron in this study.

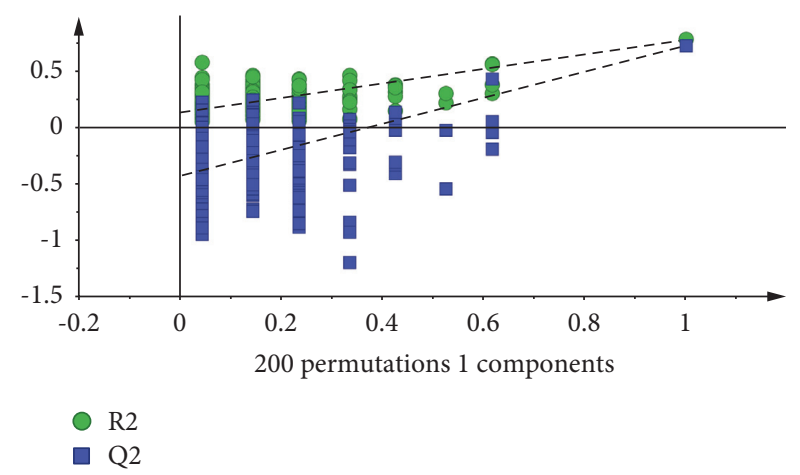

FIgURe 5: Permutation test plots of OPLS-DA model.

3.6. Quantitative Analysis of 21 Batches of Saffron. The proposed HPLC method was successfully used for simultaneous determination of crocin I, crocin II, and picrocrocin. As shown in Figure 6(a), the highest content of crocin I was from Henan $(204.97 \mathrm{mg} / \mathrm{g})$, the lowest origin was from Zhejiang $(60.06 \mathrm{mg} / \mathrm{g})$, and the median origin was from Xizang $(146.80 \mathrm{mg} / \mathrm{g})$. The highest content of crocin II was from Henan $(74.11 \mathrm{mg} / \mathrm{g})$ and the lowest origin was from Anhui $(20.10 \mathrm{mg} / \mathrm{g})$, and the median origin was from Zhejiang $(44.65 \mathrm{mg} / \mathrm{g})$. The highest content of picrocrocin was from Shanghai $(187.53 \mathrm{mg} / \mathrm{g})$, the lowest origin was from Zhejiang $(57.98 \mathrm{mg} / \mathrm{g})$, and the median origin was from Zhejiang $(110.02 \mathrm{mg} / \mathrm{g})$.

Based on the contents of the three major components, the 21 batches of saffron could be divided into two categories by HCA (Figure 6(b)): category 1 included two batches from Henan, one batch from Shanghai, three batches from Iran, one batch from Zhejiang, three batches from Xizang, and one batch from Anhui; category 2 included three batches from Zhejiang, two batches from Iran, four batches from Anhui, and one batch from Dubai.

As the results of quantitative analysis show, the contents of three major components of four batches of saffron from Zhejiang province were significantly different from each other. However, one batch of saffron from Zhejiang province was very similar to those from Tibet and Iran et al., indicating that the differences between samples had little correlation with origin. To sum up, origin was not the key factor 


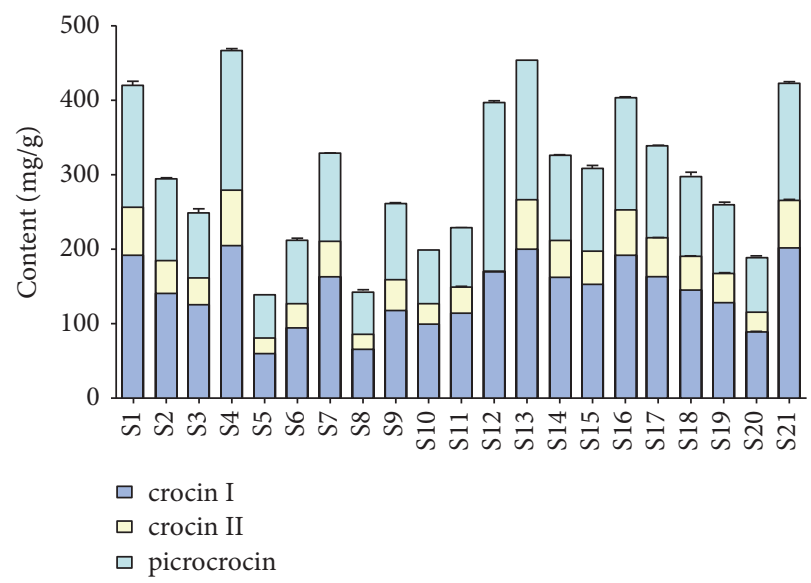

(a)

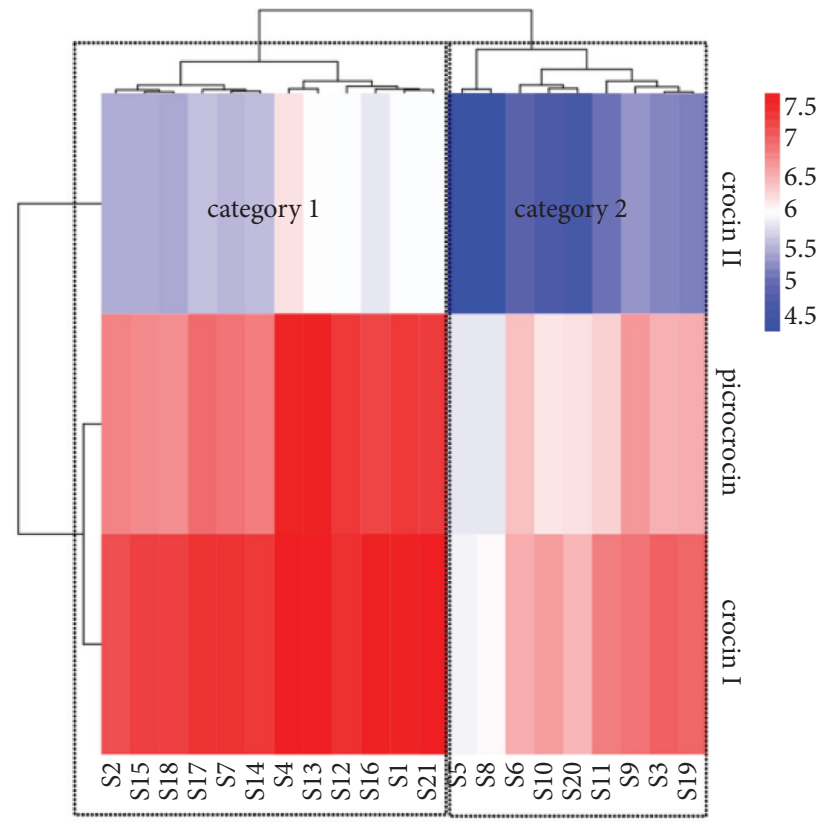

(b)

FIGURE 6: Contents of three compounds in 21 batches of saffron. Light blue, yellow, and dark blue colors represent picrocrocin, crocin I, and crocin II, respectively (a), and heat map of the HCA of three component of 21 batches of saffron (b).

of the difference that affects the quality of saffron. The difference on quality may be caused by cultivation methods, nutrition and quality of bulb, growth environment, climate, and other factors.

\subsection{Antioxidant Activities of 21 Batches of Saffron.} Oxidation is an important process for the energy productive of many biological organs [50]. Studies have shown that cancer, inflammation, blood diseases, and other diseases are closely related to oxidative free radicals, and excessive free radicals in the human body will lead to aging [51-53]. Commonly, antioxidant capacity can be assessed by in vivo and in vitro methods [54]. The in vitro methods were widely used for their advantages of simple operation, stable results, and short period. Multiple in vitro antioxidant methods have been reported in previous studies, including DPPH method, $\cdot \mathrm{OH}$ radical method, $2,2^{\prime}$-azinobis(3-ethylbenzothiazoline-6-sulfonic) acid ammonium salt (ABTS) method, and ferric reducing antioxidant potential assay (FRAP) method [55]. Because many studies focused on the pharmacological of saffron extracts (ethanolic and aqueous extracts) and saffron components (crocin and safranal), there were few literatures pertaining to antioxidant activity of saffron in vitro $[56,57]$. In this study, DPPH method and $\bullet \mathrm{OH}$ radical method were used to evaluate the antioxidant capacity of saffron. The antioxidant results in Figure 6 revealed that 21 batches of saffron exhibited a concentration dependence relationship with $\mathrm{DPPH}$ and $\bullet \mathrm{OH}$ radical-scavenging activity.

As shown in Figure $7(\mathrm{a})$, the $\mathrm{IC}_{50}$ value of DPPH radicalscavenging activity of saffron ranged from 0.66 to $2.47 \mathrm{mg} /$ $\mathrm{mL}$, which represented a variation of approximately 3.7 -fold.
The DPPH radical inhibitory capacity of the saffron decreased in the following order: $\mathrm{S} 21>\mathrm{S} 4>\mathrm{S} 7>\mathrm{S} 1>$ $\mathrm{S} 18>\mathrm{S} 12>\mathrm{S} 14>\mathrm{S} 13>\mathrm{S} 16>\mathrm{S} 17>\mathrm{S} 3>\mathrm{S} 2>\mathrm{S} 15>\mathrm{S} 8>\mathrm{S} 10$ $>\mathrm{S} 6>\mathrm{S} 19>\mathrm{S} 9>\mathrm{S} 5>\mathrm{S} 11>\mathrm{S} 20$. S21 exhibited the strongest DPPH radical-scavenging activity, followed by S4 and S7.

As shown in Figure $7(\mathrm{~b})$, the $\mathrm{IC}_{50}$ value of $\bullet \mathrm{OH}$ radicalscavenging activity of saffron ranged from 1.50 to $4.13 \mathrm{mg} /$ $\mathrm{mL}$, which represented a variation of approximately 2.8 -fold. The $\bullet \mathrm{OH}$ radical inhibitory capacity of saffron decreased in the following order: $\mathrm{S} 4>\mathrm{S} 21>\mathrm{S} 13>\mathrm{S} 12>\mathrm{S} 1>\mathrm{S} 17>\mathrm{S} 18>$ $\mathrm{S} 16>\mathrm{S} 7>\mathrm{S} 9>\mathrm{S} 14>\mathrm{S} 19>\mathrm{S} 3>\mathrm{S} 2>\mathrm{S} 11>\mathrm{S} 6>\mathrm{S} 10>\mathrm{S} 15>$ $\mathrm{S} 20>\mathrm{S} 5>\mathrm{S} 8$. S4 exhibited the strongest DPPH radicalscavenging activity, followed by S21 and S13.

As shown in Figure 7(c), two curves of $\mathrm{IC}_{50}$ values of $\mathrm{DPPH}$ and $\bullet \mathrm{OH}$ scavenging activity represented the same trend, which showed the results of antioxidant are credible.

3.8. GRA of Antioxidant Spectrum-Effect Relationship. The correlation between 21 batches of saffron and the $\mathrm{IC}_{50}$ values of DPPH radical-scavenging capacity and $\bullet \mathrm{OH}$ radical-scavenging capacity were analyzed by grey correlation degree analysis (Table 5). The contribution of 13 common peaks to the antioxidant activity depended on the correlation degree. For DPPH radical scavenging, the grey relational orders of different components in saffron were ranked as the following order: $\mathrm{P} 7>\mathrm{P} 10>\mathrm{P} 6>\mathrm{P} 3>\mathrm{P} 2>$ $\mathrm{P} 11>\mathrm{P} 9>\mathrm{P} 8>\mathrm{P} 12>\mathrm{P} 4>\mathrm{P} 13>\mathrm{P} 5>\mathrm{P} 1$. For $\bullet \mathrm{OH}$ radical scavenging, the grey relational orders of different components in saffron were ranked as the following order: $\mathrm{P} 7>\mathrm{P} 11>\mathrm{P} 10>\mathrm{P} 3>\mathrm{P} 8>\mathrm{P} 4>\mathrm{P} 6>\mathrm{P} 9>\mathrm{P} 12>\mathrm{P} 5>\mathrm{P} 1>\mathrm{P} 2$ $>\mathrm{P} 13$. The grey correlation degree of all peaks was higher 


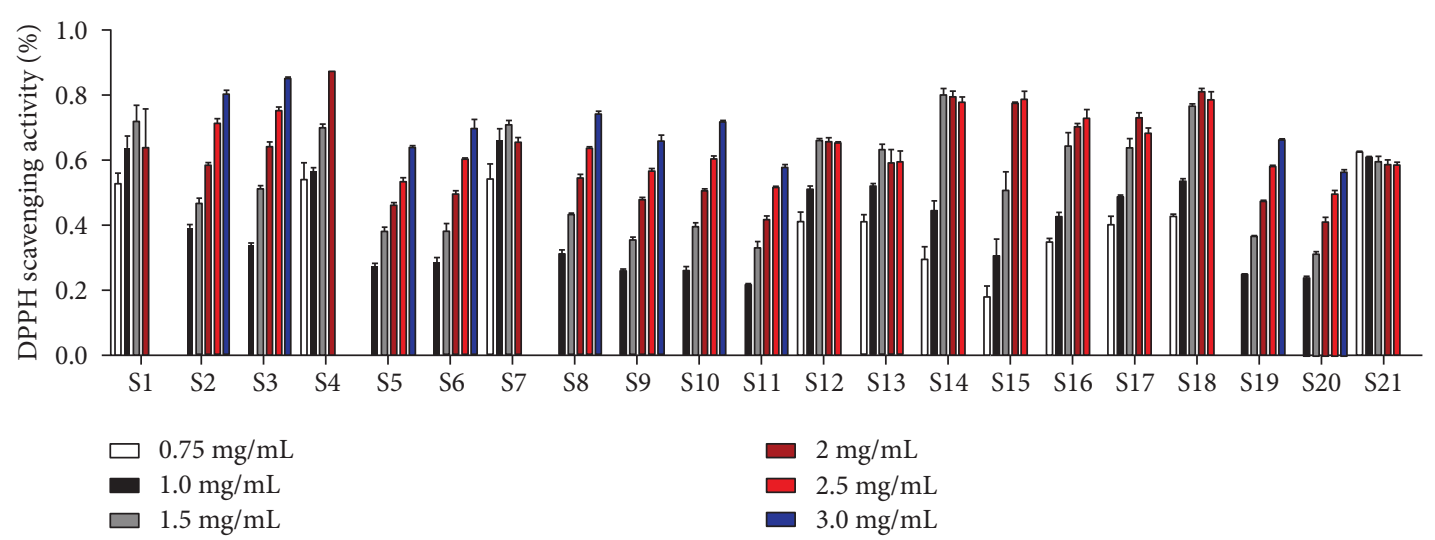

(a)

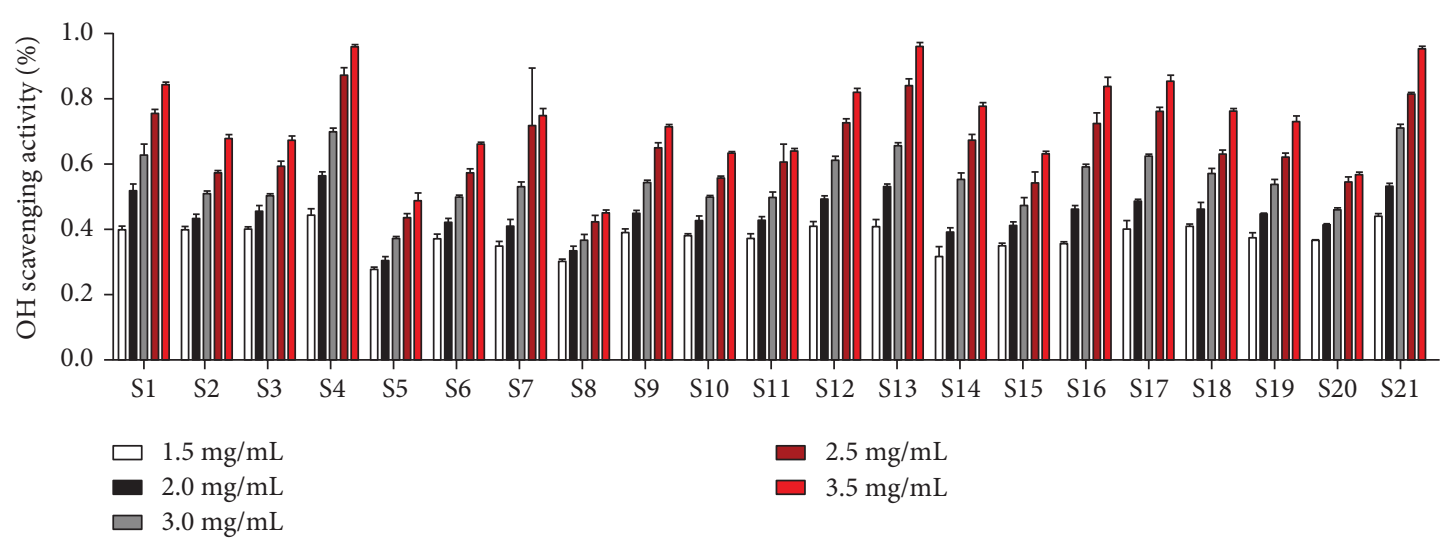

(b)

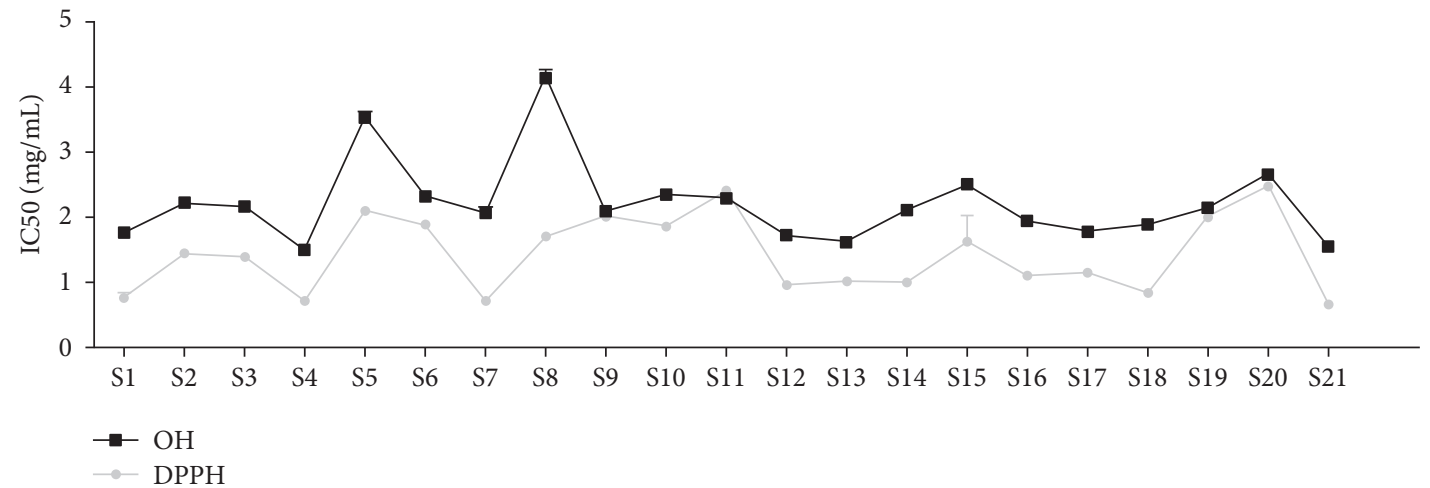

(c)

FIGURE 7: DPPH and $\bullet \mathrm{OH}$ scavenging activity of 21 batches of saffron of different concentration $(\mathrm{a}, \mathrm{b})$ and $\mathrm{IC}_{50}$ of the antioxidant activity of 21 batches of saffron (c).

TABLE 5: Results of correlation of HPLC fingerprint with antioxidant activities of saffron.

\begin{tabular}{lcr}
\hline Peak number & \multicolumn{2}{c}{ Correlation } \\
\hline 1 & DPPH & $\bullet$ OH \\
2 & 0.602 & 0.690 \\
3 & 0.736 & 0.682 \\
4 & 0.756 & 0.759 \\
5 & 0.663 & 0.727 \\
6 & 0.632 & 0.695 \\
7 & 0.760 & 0.724 \\
\hline
\end{tabular}


TABle 5: Continued.

\begin{tabular}{lcr}
\hline Peak number & & Correlation \\
\hline 8 & DPPH & $\bullet$ OH \\
9 & 0.671 & 0.753 \\
10 & 0.714 & 0.722 \\
11 & 0.772 & 0.775 \\
12 & 0.731 & 0.780 \\
13 & 0.665 & 0.715 \\
\hline
\end{tabular}

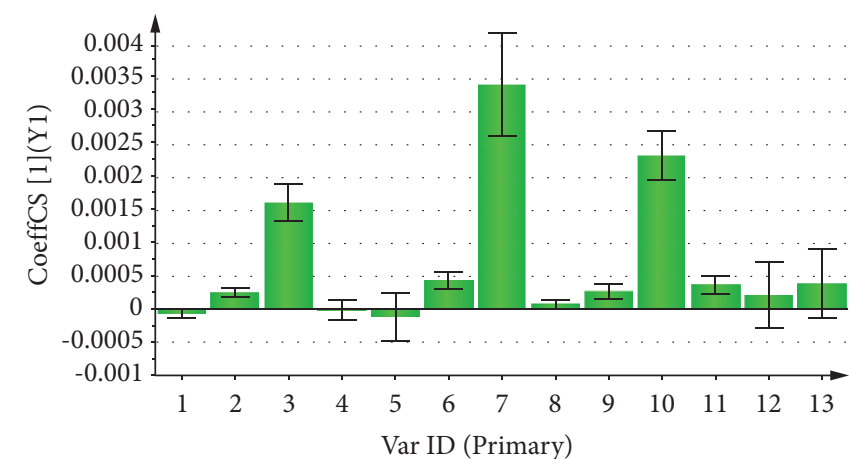

(a)

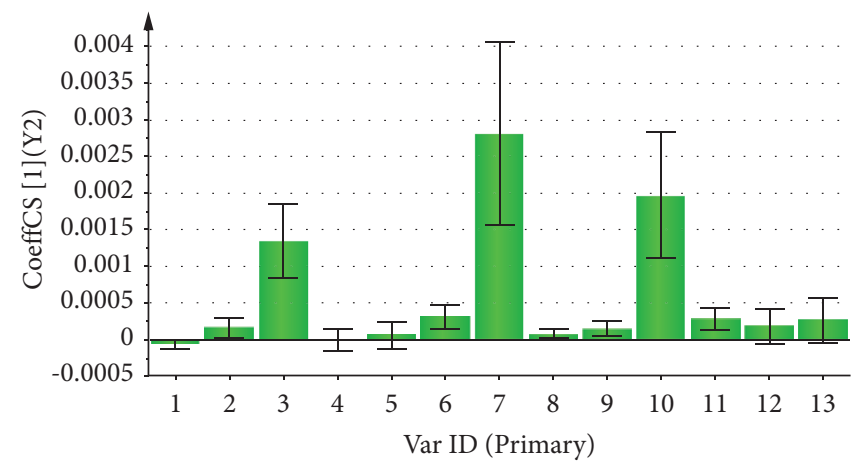

(c)

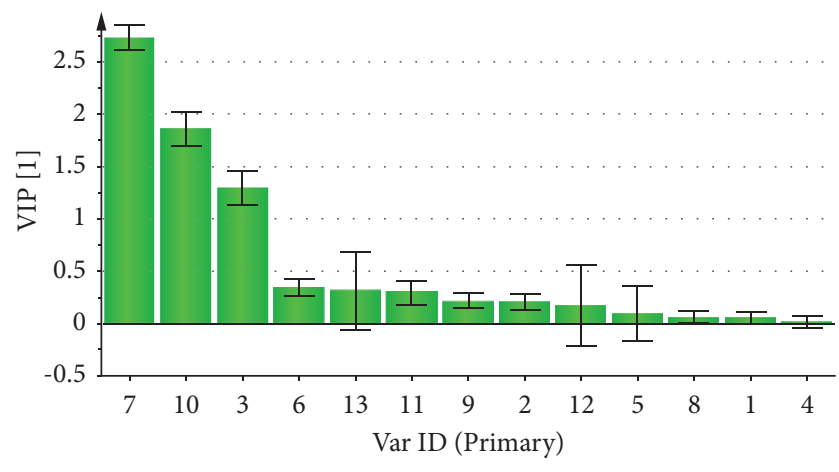

(b)

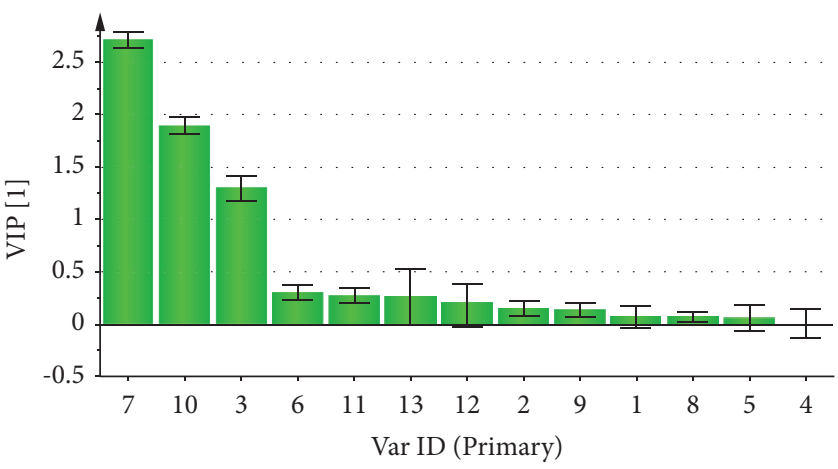

(d)

FIGURE 8: The coefficients of the partial least squares regression analysis of DPPH and $\bullet \mathrm{OH}$ radical-scavenging capacity (a, c) and the VIP values of the partial least squares regression analysis of $\mathrm{DPPH}$ and $\bullet \mathrm{OH}$ radical-scavenging capacity $(\mathrm{b}, \mathrm{d})$.

than 0.6 , which indicated that the antioxidant activity was caused by common functions of several components [32].

3.9. PLS of Antioxidant Spectrum-Effect Relationship. The PA of 13 common peaks of 21 batches of saffron was inputted as the independent variable, while the IC50 of DPPH and $\bullet \mathrm{OH}$ radical-scavenging capacity were inputted as the dependent variable, and the partial least squares method was used to analyze the variables. The correlation coefficient and variable projection importance value (VIP) were obtained to evaluate the correlation between 13 chromatographic peaks and drug efficacy and their contribution to drug efficacy. It is generally believed that when VIP $>1$, the independent variable has significant importance on explaining the dependent variable. In this study, the VIP value of each chromatographic peak of $\mathrm{DPPH}$ radical-scavenging capacity is ranked as follows: P7 > P10 > P3 > P6 > P13 > P11 > P9 >
P2 $>$ P12 $>$ P5 $>$ P8 $>$ P1 > P4 (Figures 8(a) and 8(b)). The VIP value of each chromatographic peak of $\bullet \mathrm{OH}$ radical-scavenging capacity is ranked as follows: $\mathrm{P} 7>\mathrm{P} 10>\mathrm{P} 3>\mathrm{P} 6>\mathrm{P} 11>$ $\mathrm{P} 13>\mathrm{P} 12>\mathrm{P} 2>\mathrm{P} 9>\mathrm{P} 1>\mathrm{P} 8>\mathrm{P} 5>\mathrm{P} 4$ (Figures 8(c) and $8(\mathrm{~d})$ ). It can be seen that the VIP values of P7 (crocin I), P10 (crocin II), and P3 (picrocrocin) were higher than 1, indicating that these compounds could be the core components of antioxidant activity in saffron.

Limited relevant research showed that polysaccharide and ethanol extracts of saffron from seven different productions had remarkable antioxidant activities [58]. Further research showed that both stigmas and flowers had antioxidant capacity, due to the apocarotenoids, flavonoids, and flavonols presented in the stigmas and the flavonoids, antocyanins, and tannins abundant in the remaining portions of $C$. sativus flower. However, the exact mechanisms and compounds responsible for the antioxidant activity of saffron were still not clear. Based on the results in this study, the grey correlation 


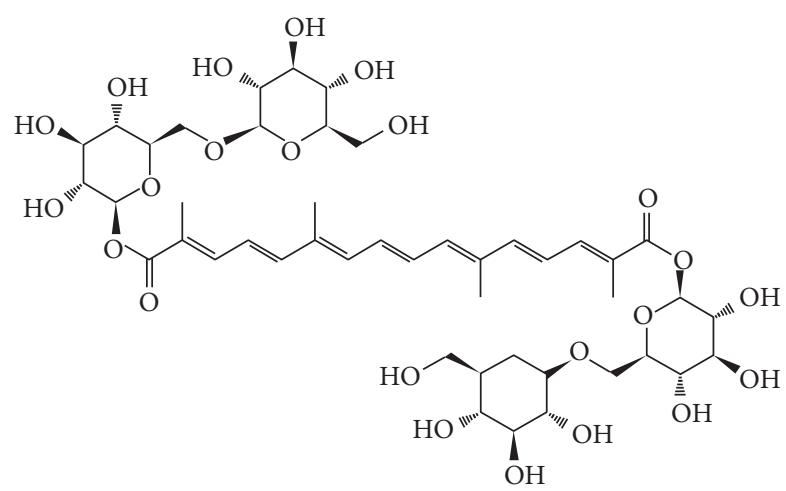

(a)

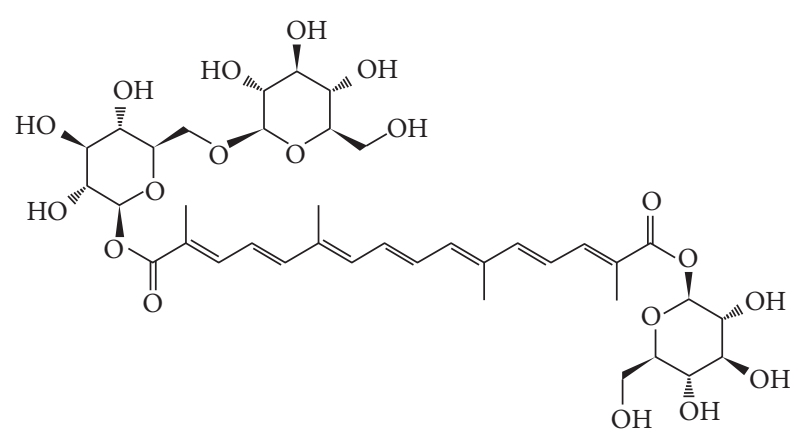

(b)<smiles>CC1=C(C=O)C(C)(C)CC(OC2OC(CO)C(O)C(O)C2O)C1</smiles>

(c)

FIgURE 9: The chemical structure of crocin I (a), crocin II (b), and picrocrocin (c).

degree and OPLS-DA of antioxidant spectrum-effect relationship showed crocin I, crocin II, and picrocrocin had large contribution coefficients, closely related to antioxidant ability. Many studies have shown that water-soluble carotenoids show excellent antioxidant potential, which was related to the conjugated large $\pi$ bond in the molecular structure of carotenoids and easily react with free radicals to form harmless products or scavenge-free radicals by destroying free radical chains. Crocins (Figure 9) are a group of water soluble-carotenoids, produced by zeaxanthin through enzymatic cleavage [59]. Two of the most abundant crocin were crocin I (trans-crocetin di-( $\beta$-d-gentiobiosyl) ester) and crocin II (crocetin-( $\beta$-d-glucosyl)-( $\beta$-gentibiosyl) ester) [60]. It can be inferred that the antioxidant capacity of these two components is closely related to their chemical structures and higher content in saffron [58]. In this study, picrocrocin was demonstrated to be directly related to antioxidant activity for the first time. Crocin I, crocin II, and picrocrocin accounted for a large proportion of contents in saffron and presented the good antioxidant ability, which made saffron a good candidate for an antioxidant food.

Previous studies have focused on mainly the analysis of its chemical composition or only bioactivities, limiting the development and utilization of saffron as a commercial medicine $[61,62]$. In this study, the correlation between chemical components and pharmacological activity of saffron were analyzed by spectrum-effect relationship. Crocin I, crocin II, and picrocrocin were identified to be closely related to antioxidant capacity, which were conducive to the establishment of a more reliable and scientific quality standard for saffron. However, the drawback of the current study was that only 21 batches of samples were used in this study, which was not conducive to establishing a firm correlation. In order to confirm the findings of this research, a larger sample size should be considered in the future.

\section{Conclusion}

The spectrum-effect relationships of HPLC fingerprints and scavenging capacity for $\mathrm{DPPH}$ and $\bullet \mathrm{OH}$ were established to analyze the active components of saffron. The spectrumeffect relationships on the basis of grey correlation degree and OPLS-DA analysis revealed that crocin I, crocin II, and picrocrocin were the main components contributing to the antioxidant activities of saffron and these compounds had synergistic antioxidant effects. Through this study, the main antioxidant components of saffron were further determined, which could provide clue for establishing reliable and reasonable quality standards for saffron and its products.

\section{Data Availability}

The data used to support the findings of this study are included within the article.

\section{Disclosure}

Ya You and Zijin Xu are co-first authors.

\section{Conflicts of Interest}

The authors have no conflicts of interest to declare.

\section{Authors' Contributions}

Ya You, Zijin Xu, Suhong Chen, and Ping Wang contributed equally to this work. Ping Wang, Suhong Chen, and Yifeng Cao conceived of and designed the experiments; Ya You, Zijin $\mathrm{Xu}$, Qingrou Zhong, and Lin Zhu performed the experiments; YaYou, Yi Tao, Susu Lin, and Qiaoqiao Li analyzed the data; Ya You wrote the paper. 


\section{Acknowledgments}

Thanks are due to the Special Project of International Technology Cooperation of One Belt and One Road (grant/ award number: 2017C04009) and the Key Projects of International Scientific and Technological Innovation Cooperation between Governments (grant/award number: 2017YFE0130100).

\section{References}

[1] M. Asadollahi, P. Nikdokht, B. Hatef et al., "Protective properties of the aqueous extract of saffron (Crocus sativus L.) in ischemic stroke, randomized clinical trial," Journal of Ethnopharmacology, vol. 238, no. 1, Article ID 111833, 2019.

[2] S. Lin, Q. Li, S. Jiang et al., "Crocetin ameliorates chronic restraint stress-induced depression-like behaviors in mice by regulating MEK/ERK pathways and gut microbiota," Journal of Ethnopharmacology, vol. 268, Article ID 113608, 2021.

[3] A. Lambrianidou, F. Koutsougianni, I. Papapostolou, and K. Dimas, "Recent advances on the anticancer properties of saffron (Crocus sativus L.) and its major constituents," Molecules, vol. 26, no. 1, p. 86, 2020.

[4] M. Ghasemnejad-Berenji, "Immunomodulatory and antiinflammatory potential of crocin in COVID-19 treatment," Journal of Food Biochemistry, vol. 45, no. 5, Article ID e13718, 2021.

[5] S. Faridi, N. Delirezh, and S. M. Abtahi Froushani, "Beneficial effects of hydroalcoholic extract of saffron in alleviating experimental autoimmune diabetes in $\mathrm{C} 57 \mathrm{bl} / 6$ mice," Iranian Journal of Allergy, Asthma and Immunology, vol. 18, no. 1, pp. 38-47, 2019.

[6] A. Mokhtari-Zaer, S. Saadat, V. Ghorani, A. Memarzia, and M. H. Boskabady, "The effects of saffron (Crocus sativus) and its constituents on immune system," in Saffron, pp. 193-217, Elsevier, Amsterdam, Netherlands, 2020.

[7] Z. Xu, S. Lin, J. Gong et al., "Exploring the protective effects and mechanism of crocetin from saffron against NAFLD by network pharmacology and experimental validation," Frontiers in Medicine, vol. 8, Article ID 681391, 2021.

[8] A. Amirvaresi, N. Nikounezhad, M. Amirahmadi, B. Daraei, and H. Parastar, "Comparison of near-infrared (NIR) and mid-infrared (MIR) spectroscopy based on chemometrics for saffron authentication and adulteration detection," Food Chemistry, vol. 344, no. 33, Article ID 128647, 2020.

[9] I. Bosmali, S. A. Ordoudi, M. Z. Tsimidou, and P. Madesis, "Greek PDO saffron authentication studies using species specific molecular markers," Food Research International, vol. 100, no. 1, pp. 899-907, 2017.

[10] C. N. Reddy, S. B. Bharate, R. A. Vishwakarma, and S. S. Bharate, "Chemical analysis of saffron by HPLC based crocetin estimation," Journal of Pharmaceutical and Biomedical Analysis, vol. 181, Article ID 113094, 2020.

[11] W.-J. Huang, F.-F. Li, Y.-J. Liu, and C.-L. Long, "Identification of crocus sativus and its adulterants from Chinese markets by using DNA barcoding technique," Iranian Journal of Biotechnology, vol. 13, no. 1, pp. 36-42, 2015.

[12] A. Zalacain, S. A. Ordoudi, I. Blázquez et al., "Screening method for the detection of artificial colours in saffron using derivative UV-Vis spectrometry after precipitation of crocetin," Food Additives and Contaminants, vol. 22, no. 77, pp. 607-15, 2005.

[13] V. Ashok, N. Agrawal, J. Esteve-Romero, D. Bose, and N. P. Dubey, "Detection of methyl orange in saffron and other edibles using direct injection micellar liquid chromatography," Food Analytical Methods, vol. 10, no. 1, pp. 269-276, 2017.

[14] A. M. Sánchez, L. Maggi, M. Carmona, and G. L. Alonso, "Authentication of saffron spice (Crocus sativus L.)," ACS Symposium Series, vol. 1081, pp. 309-331, 2011.

[15] E. A. Petrakis and M. G. Polissiou, "Assessing saffron (Crocus sativus L.) adulteration with plant-derived adulterants by diffuse reflectance infrared Fourier transform spectroscopy coupled with chemometrics," Talanta, vol. 162, pp. 558-566, 2017.

[16] H. Dai, Q. Gao, and L. He, "Rapid determination of saffron grade and adulteration by thin-layer chromatography coupled with Raman spectroscopy," Food Analytical Methods, vol. 13, no. 11, pp. 2128-2137, 2020.

[17] M. Bononi, P. Milella, and F. Tateo, "Gas chromatography of safranal as preferable method for the commercial grading of saffron (Crocus sativus L.)," Food Chemistry, vol. 176, pp. 17-21, 2015.

[18] L. Sabatino, M. Scordino, M. Gargano, A. Belligno, P. Traulo, and G. Gagliano, "HPLC/PDA/ESI-MS evaluation of saffron (Crocus sativus L.) adulteration," Natural Product Communications, vol. 6, no. 12, pp. 1873-6, 2011.

[19] Y. Tong, X. Zhu, Y. Yan et al., "The influence of different drying methods on constituents and antioxidant activity of saffron from China," International journal of Analytical Chemistry, vol. 2015, no. 1, Article ID 953164, 2015.

[20] E. Anastasaki, C. Kanakis, C. Pappas et al., "Geographical differentiation of saffron by GC-MS/FID and chemometrics," European Food Research and Technology, vol. 229, no. 6, pp. 899-905, 2009.

[21] J. Liu, N. Chen, J. Yang et al., "An integrated approach combining HPLC, GC/MS, NIRS, and chemometrics for the geographical discrimination and commercial categorization of saffron," Food Chemistry, vol. 253, pp. 284-292, 2018.

[22] R. Rocchi, M. Mascini, M. Sergi, D. Compagnone, D. Mastrocola, and P. Pittia, "Crocins pattern in saffron detected by UHPLC-MS/MS as marker of quality, process and traceability," Food Chemistry, vol. 264, pp. 241-249, 2018.

[23] R. Rocchi, M. Mascini, F. Angelo et al., "Comparison of IRMS, GC-MS and E-Nose data for the discrimination of saffron samples with different origin, process and age," Food Control, vol. 106, Article ID 106736, 2019.

[24] S. Zoughi, F. Faridbod, A. Amiri, and M. R. Ganjali, "Detection of tartrazine in fake saffron containing products by a sensitive optical nanosensor," Food Chemistry, vol. 350, no. 5, Article ID 129197, 2021.

[25] F. D. Donato, A. A. D’Archivio, M. A. Maggi, and L. Rossi, "Detection of plant-derived adulterants in saffron (Crocus sativus L.) by HS-spme/GC-MS profiling of volatiles and chemometrics," Food Analytical Methods, vol. 14, no. 4, pp. 1-13, 2021.

[26] M. Masár, J. Hradski, A. Mikovíková, and R. Szucs, “Determination of carminic acid in foodstuffs and pharmaceuticals by microchip electrophoresis with photometric detection," Separations, vol. 7, no. 4, p. 72, 2020.

[27] P. Morozzi, A. Zappi, F. Gottardi, M. Locatelli, and D. Melucci, "A quick and efficient non-targeted screening test for saffron authentication: application of chemometrics to gas-chromatographic data," Molecules, vol. 24, no. 14, p. 2602, 2019.

[28] X.-F. Zhang, J. Chen, J.-L. Yang, and Y.-P. Shi, "UPLC-MS/ MS analysis for antioxidant components of Lycii Fructus 
based on spectrum-effect relationship," Talanta, vol. 180, pp. 389-395, 2017.

[29] H. Liu, S. Zhu, Q. Liu, and Y. Zhang, "Spectrum-effect relationship study between HPLC fingerprints and antioxidant of honeysuckle extract," Biomedical Chromatography, vol. 33, no. 10, p. e4583, 2019.

[30] Q. Fan, R. Yang, F. Yang, P. Xia, and L. Zhao, "Spectrum-effect relationship between HPLC fingerprints and antioxidant activity of Angelica sinensis," Biomedical Chromatography, vol. 34, no. 2, p. e4707, 2020.

[31] Z. Shi, Z. Liu, C. Liu et al., "Spectrum-effect relationships between chemical fingerprints and antibacterial effects of Lonicerae Japonicae Flos and Lonicerae Flos base on UPLC and microcalorimetry," Frontiers in Pharmacology, vol. 7, p. 12, 2016.

[32] X. Bai, L. Liu, J. Zhang et al., "Spectrum-effect relationship between GC-Q-TOF-MS fingerprint and antioxidant, antiinflammatory activities of Schizonepeta tenuifolia essential oil," Biomedical Chromatography, vol. 35, no. 1, p. e5106, 2021.

[33] M. Kabiri, H. Rezadoost, and A. Ghassempour, "A comparative quality study of saffron constituents through HPLC and HPTLC methods followed by isolation of crocins and picrocrocin," Food Science and Technology, vol. 84, pp. 1-9, 2017.

[34] C. Wu, B. Xu, Z. Li, P. Song, and Z. Chao, "Gender discrimination of Populus tomentosa barks by HPLC fingerprint combined with multivariate statistics," Plant Direct, vol. 5, no. 3, Article ID e00311, 2021.

[35] D. Gong, J. Chen, Y. Sun, X. Liu, and G.-Y. Sun, "Multiple wavelengths maximization fusion fingerprint profiling for quality evaluation of compound liquorice tablets and related antioxidant activity analysis," Microchemical Journal, vol. 160, Article ID 105671, 2021.

[36] L. Wang, X. Ren, Y. Wang et al., "HPLC fingerprint and UV-Vis spectroscopy coupled with chemometrics for Curcumae radix species discrimination and three bioactive compounds prediction," Microchemical Journal, vol. 166, Article ID 106254, 2015.

[37] L. Jian, L. Y. Zhou, J. F. Bi, X. Liu, and X. Y. Wu, "Quality evaluation of yellow peach chips prepared by explosion puffing drying," Journal of Food Science \& Technology, vol. 52, no. 12, pp. 8204-8211, 2015.

[38] L. Chen, F. Yu, S. Sun, X. Liu, and C. Xue, "Evaluation indicators of Ruditapes philippinarum nutritional quality," Journal of Food Science \& Technology, vol. 58, no. 1, pp. 2943-2951, 2020.

[39] M. Najafi, A. Leufven, M. Dovom, N. Sedaghat, and A. Pourfarzad, "Probing the interactions between hardness and sensory of pistachio nuts during storage using principal component analysis," Food Sciences and Nutrition, vol. 7, no. 8, pp. 2684-2691, 2019.

[40] B. M. Huang, Q. L. Zha, T. B. Chen, S. Y. Xiao, and H. Zhou, "Discovery of markers for discriminating the age of cultivated ginseng by using UHPLC-QTOF/MS coupled with OPLSDA," Phytomedicine, vol. 45, 2018.

[41] M. Bylesjö, M. Rantalainen, O. Cloarec, J. K. Nicholson, E. Holmes, and J. Trygg, "OPLS discriminant analysis: combining the strengths of PLS-DA and SIMCA classification," Journal of Chemometrics, vol. 20, no. 8-10, pp. 341-351, 2006.

[42] Y. Zheng, X. Zeng, P. Wei, W. Zhong, and W. Su, "Study on the discrimination between citri reticulatae pericarpium varieties based on HS-SPME-GC-MS combined with multivariate statistical analyses," Molecules, vol. 23, no. 5, p. 1235, 2018.

[43] H. Ishwaran and M. Lu, "Standard errors and confidence intervals for variable importance in random forest regression, classification, and survival," Statistics in Medicine, vol. 38, 2018.

[44] G. Lee and K. Lee, "Feature selection using distributions of orthogonal PLS regression vectors in spectral data," BioData Mining, vol. 14, no. 1, 2021.

[45] J. K. Ahn, J. Kim, J. Hwang, J. Song, K. H. Kim, and H.-S. Cha, "Potential metabolomic biomarkers for reliable diagnosis of Behcet's disease using gas chromatography/time-of-flightmass spectrometry," Joint Bone Spine, vol. 85, no. 3, pp. $337-343,2018$

[46] M. N. Triba, L. L. Moyec, R. Amathieu et al., "PLS/OPLS models in metabolomics: the impact of permutation of dataset rows on the K-fold cross-validation quality parameters," Molecular BioSystems, vol. 11, no. 1, pp. 13-19, 2014.

[47] M. Stranska, L. Uttl, K. Bechynska, K. Hurkova, B. Adam, and H. Jana, "Metabolomic fingerprinting as a tool for authentication of grapevine (Vitis vinifera L.) biomass used in food production," Food Chemistry, vol. 361, Article ID 130166, 2021.

[48] S. Li, Q. Shao, C. Duan, and H. Yi, "Rapid determination of crocins in saffron by near-infrared spectroscopy combined with chemometric techniques," Spectrochimica Acta Part A: Molecular and Biomolecular Spectroscopy, vol. 190, pp. 283-289, 2018.

[49] S. I. Bukhari, M. Manzoor, and M. K. Dhar, "A comprehensive review of the pharmacological potential of Crocus sativus and its bioactive apocarotenoids," Biomedicine \& Pharmacotherapy, vol. 98, pp. 733-745, 2018.

[50] J. Chen, J. Gao, and S. Guoxiang, "Quantitative analysis combined with chromatographic fingerprint and antioxidant activities for the comprehensive evaluation of compound danshen tablets," Journal of Separation Science, vol. 40, no. 6, pp. 1244-1253, 2017.

[51] J. Li, S. Wang, J. Duan et al., "The protective mechanism of resveratrol against hepatic injury induced by iron overload in mice," Toxicology and Applied Pharmacology, vol. 424, Article ID 115596, 2021.

[52] S. A. Raza and T. Touqeer, "Anti-obesity attributes; UHPLCQTOF-MS/MS-based metabolite profiling and molecular docking insights of Taraxacum officinale," Molecules Online, vol. 25, no. 21, p. 4935, 2020.

[53] E. Cecerska-Heryć, O. Surowska, R. Heryć, N. Serwin, S. Napiontek-Balińska, and B. Dołęgowska, "Are antioxidant enzymes essential markers in the diagnosis and monitoring of cancer patients-a review," Clinical Biochemistry, vol. 93, pp. 1-8, 2021.

[54] E. Niki, "Assessment of antioxidant capacity in vitro and in vivo," Free Radical Biology and Medicine, vol. 49, no. 4, pp. 503-515, 2010.

[55] M. Agrawal, S. Bansal, and K. Chopra, "Evaluation of the in vitro and in vivo antioxidant potentials of food grade Phycocyanin," Journal of Food Science \& Technology, vol. 58, pp. 4382-4390, 2021.

[56] H. Hosseinzadeh and H. M. Younesi, "Antinociceptive and anti-inflammatory effects of Crocus sativus L. stigma and petal extracts in mice," BMC Pharmacology, vol. 2, no. 1, p. 7, 2002.

[57] A. N. Assimopoulou, Z. Sinakos, and V. P. Papageorgiou, "Radical scavenging activity of Crocus sativus L. extract and its bioactive constituents," Phytotherapy Research, vol. 19, no. 11, pp. 997-1000, 2005. 
[58] A. Zhang, Y. Shen, M. Cen et al., "Polysaccharide and crocin contents, and antioxidant activity of saffron from different origins," Industrial Crops and Products, vol. 133, pp. 111-117, 2019.

[59] M. J. Gregory, R. C. Menary, and N. W. Davies, "Effect of drying temperature and air flow on the production and retention of secondary metabolites in saffron," Journal of Agricultural and Food Chemistry, vol. 53, no. 15, pp. 5969-5975, 2005.

[60] E. Mirhadi, H. Nassirli, and B. Malaekeh-Nikouei, "An updated review on therapeutic effects of nanoparticle-based formulations of saffron components (safranal, crocin, and crocetin)," Journal of Pharmaceutical Investigation, vol. 50, no. 1, pp. 47-58, 2019.

[61] V. M. Popa, C. Bele, M. A. Poiana, D. Dumbrava, and C. Jianu, "Evaluation of bioactive compounds and of antioxidant properties in some oils obtained from food industry byproducts," Romanian Biotechnological Letters, vol. 16, no. 3, pp. 6234-6241, 2011.

[62] S. Ghaffari and N. Roshanravan, "Saffron; an updated review on biological properties with special focus on cardiovascular effects," Biomedicine \& Pharmacotherapy, vol. 109, pp. 21-27, 2019. 